\title{
Symptomatic menopausal transition and risk of subsequent stroke
}

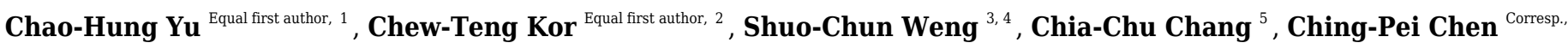 \\ 1 , Chia-Lin Wu ${ }^{\text {Corresp. } 2,4,5}$ \\ 1 Division of Cardiology, Department of Internal Medicine, Changhua Christian Hospital, Changhua, Taiwan \\ 2 Internal Medicine Research Center, Changhua Christian Hospital, Changhua, Taiwan \\ ${ }^{3}$ Center for Geriatrics and Gerontology, Department of Internal Medicine, Taichung Veterans General Hospital, Taichung, Taiwan \\ 4 Institute of Clinical Medicine, National Yang-Ming University, Taipei, Taiwan \\ 5 Division of Nephrology, Department of Internal Medicine, Changhua Christian Hospital, Changhua, Taiwan \\ Corresponding Authors: Ching-Pei Chen, Chia-Lin Wu \\ Email address: chingpei@cch.org.tw, 143843@cch.org.tw
}

Objective. To examine the long-term risk of stroke in women who have experienced symptomatic menopausal transition. Methods. In this nationwide, population-based cohort study conducted from January 1, 2000 to December 31, 2013, we identified 22058 women with no prior history of stroke, who experienced symptomatic menopausal transition at $\geq 45$ years of age. Moreover, 22058 women without symptomatic menopause were matched by propensity scores and enrolled as a comparison group. The propensity score was calculated by using all characteristic variables of each subject, including demographics (age and monthly income), comorbidities (hypertension, hyperlipidemia, diabetes mellitus, obesity, chronic kidney disease, coronary artery disease, congestive heart failure, chronic obstructive pulmonary disease, dysrhythmia, peripheral artery occlusive disease), Charlson's comorbidity index score, clinic visit frequency, and longterm medications (antihypertensives, antidiabetic agents, statins, antiplatelets, aspirin, warfarin, and hormone replacement therapy). The primary endpoint was the development of stroke after the onset of symptomatic menopausal transition. The Fine and Gray's proportional subhazards model was performed to assess the association between symptomatic menopausal transition and subsequent stroke. All subjects were followed up until December 31, 2013. Results. During a mean follow-up of 8.5 years (standard deviation 4.7 years, maximum 14 years), 2274 (10.31\%) women with symptomatic menopausal transition and 1184 (5.37\%) matched comparison participants developed stroke. The incidence rates were 11.17 per 1000 person-years in the symptomatic menopausal transition group compared with 8.57 per 1000 person-years in the comparison group. The risk of developing stroke was significantly higher in women with symptomatic menopausal transition (crude subhazard ratio, 1.31; 95\% confidence interval, 1.22 to 1.41; 
$P<0.001$ ). After adjusting for demographics, comorbidities, clinic visit frequency, and long-term medications, the risk of stroke remained statistically significant (adjusted subhazard ratio, 1.30; 95\% confidence interval, 1.21-1.40; $P<0.001$ ). Moreover, subgroup analyses revealed no evidence for inconsistent effects for symptomatic menopausal transition on subsequent risk of stroke across all subgroups except age, comorbidities, hypertension, and use of antihypertensives. Women with early menopausal transition (before age 50), without comorbid condition, without hypertension, or without use of antihypertensives are at a higher risk of stroke. The longer duration of symptomatic menopausal transition was associated with higher risk of stroke $(P$ for trend $<0.001)$. Conclusion. In this large-scale retrospective cohort study, symptomatic menopausal transition was statistically significantly associated with a $30 \%$ increased risk of stroke. Further prospective studies are required to confirm our findings. 


\section{Symptomatic Menopausal Transition and Risk of Subsequent}

\section{Stroke}

3 Chao-Hung $\mathrm{Yu}^{1}$, Chew-Teng Kor ${ }^{2}$, Shuo-Chun Weng ${ }^{3,4}$, Chia-Chu Chang ${ }^{5}$, Ching-Pei Chen ${ }^{1}$, 4 and Chia-Lin $\mathrm{Wu}^{2,4,5}$

5

6 'Division of Cardiology, Department of Internal Medicine, Changhua Christian Hospital,

7 Changhua, Taiwan

8 Internal Medicine Research Center, Changhua Christian Hospital, Changhua, Taiwan

$9{ }^{3}$ Center for Geriatrics and Gerontology, Department of Internal Medicine, Taichung Veterans

10 General Hospital, Taichung, Taiwan

11 Institute of Clinical Medicine, National Yang-Ming University, Taipei, Taiwan

$12{ }^{5}$ Division of Nephrology, Department of Internal Medicine, Changhua Christian Hospital,

13 Changhua, Taiwan

14

15

Drs. Yu and Kor contributed equally to this work.

16

17 Corresponding Authors:

18 Ching-Pei Chen (E-mail: chingpei@cch.org.tw), Division of Cardiology or

19 Chia-Lin Wu (E-mail: 143843@cch.org.tw), Division of Nephrology,

20 Department of Internal Medicine,

21 Changhua Christian Hospital,

22 No. 135, Nan-Siau Street,

23 Changhua,

24 Taiwan 500. 


\section{Abstract}

26 Objective. To examine the long-term risk of stroke in women who have experienced

27 symptomatic menopausal transition.

28 Methods. In this nationwide, population-based cohort study conducted from January 1, 2000 to

29 December 31, 2013, we identified 22058 women with no prior history of stroke, who

30 experienced symptomatic menopausal transition at $\geq 45$ years of age. Moreover, 22058 women

31 without symptomatic menopause were matched by propensity scores and enrolled as a

32 comparison group. The propensity score was calculated by using all characteristic variables of

33 each subject, including demographics (age and monthly income), comorbidities (hypertension,

34 hyperlipidemia, diabetes mellitus, obesity, chronic kidney disease, coronary artery disease,

35 congestive heart failure, chronic obstructive pulmonary disease, dysrhythmia, peripheral artery

36 occlusive disease), Charlson's comorbidity index score, clinic visit frequency, and long-term

37 medications (antihypertensives, antidiabetic agents, statins, antiplatelets, aspirin, warfarin, and

38 hormone replacement therapy). The primary endpoint was the development of stroke after the

39 onset of symptomatic menopausal transition. The Fine and Gray's proportional subhazards

40 model was performed to assess the association between symptomatic menopausal transition and

41 subsequent stroke. All subjects were followed up until December 31, 2013.

42 Results. During a mean follow-up of 8.5 years (standard deviation 4.7 years, maximum 14 
43 years), $2274(10.31 \%)$ women with symptomatic menopausal transition and 1184 (5.37\%)

44 matched comparison participants developed stroke. The incidence rates were 11.17 per 1000

45 person-years in the symptomatic menopausal transition group compared with 8.57 per 1000

46 person-years in the comparison group. The risk of developing stroke was significantly higher in

47 women with symptomatic menopausal transition (crude subhazard ratio, $1.31 ; 95 \%$ confidence

48 interval, 1.22 to $1.41 ; P<0.001)$. After adjusting for demographics, comorbidities, clinic visit

49 frequency, and long-term medications, the risk of stroke remained statistically significant

50 (adjusted subhazard ratio, 1.30; 95\% confidence interval, 1.21-1.40; $P<0.001$ ). Moreover,

51 subgroup analyses revealed no evidence for inconsistent effects for symptomatic menopausal

52 transition on subsequent risk of stroke across all subgroups except age, comorbidities,

53 hypertension, and use of antihypertensives. Women with early menopausal transition (before age

54 50), without comorbid condition, without hypertension, or without use of antihypertensives are at

55 a higher risk of stroke. The longer duration of symptomatic menopausal transition was associated

56 with higher risk of stroke $(P$ for trend $<0.001)$.

57 Conclusion. In this large-scale retrospective cohort study, symptomatic menopausal transition

58 was statistically significantly associated with a 30\% increased risk of stroke. Further prospective

59 studies are required to confirm our findings.

60 Keywords: menopause, stroke, transition, women 
61 


\section{Introduction}

63 Menopausal transition is defined as the period before the final menstrual period in late

64 reproductive life. Menopause occurs following 12 months of amenorrhea and represents the loss

65 of ovarian follicular function; this typically occurs in women between 45 and 55 years of age.

66 Approximately 1.5 million women experience menopausal transition every year in the United

67 States (U.S. Census B., 2005). Vasomotor symptoms, insomnia, depression, and vaginal dryness

68 are major problems during menopausal transition (Santoro, Epperson, \& Mathews, 2015).

69 Stroke is the second leading cause of death and third leading cause of disability-adjusted life-

70 years lost worldwide. After stroke, the survivors still have high rates of mortality, recurrent

71 stroke, and disability for months to years (Hankey, 2017). The Chinese populations in China and

72 Taiwan were reported to have higher stroke incidence (age-standardized annual first-ever stroke

73 incidence: range $205-584$ per 100,000 patients) than white populations (range 170-335 per

74 100,000 patients) (Tsai, 2013). Thus, stroke prevention for populations at high risk for stroke

75 remains an important issue in Taiwan.

76 Symptoms of menopausal transition, such as vasomotor symptoms and night sweats, may

77 have a negative impact on a woman's quality of life during her midlife (Thurston, et al., 2012).

78 The occurrence of vasomotor symptoms is associated with low serum estrogen levels (Erlik,

79 Meldrum, \& Judd, 1982). In addition, vasomotor symptoms have been found to be associated 
80 with chronic insomnia (Ohayon, 2006), cardiovascular disease (Rossouw, et al., 2007), and

81 insulin resistance (Huang, et al., 2017). A growing body of evidence has also shown the

82 association of vasomotor symptoms with poor control of dyslipidemia in obese women

83 (Thurston, et al., 2012), atherosclerosis, vascular endothelial dysfunction (Thurston, et al., 2017),

84 increased prothrombotic events, smoking habits (Gallicchio, et al., 2014 ), and disturbed

85 sympathovagal tone (Thurston, et al., 2012; Gallicchio, et al., 2014). These deleterious effects

86 accompanied by the symptomatic menopausal transition may potentially contribute to stroke as

87 well as cardiovascular disease. However, no previous studies have assessed the direct link

88 between symptomatic menopausal transition and the risk of stroke with a long-term follow-up.

89 We hypothesized that symptomatic menopausal transition may have insidious adverse effects on

90 the cerebrovascular system.

91 Therefore, we conducted this nationwide, large-scale, population-based cohort study to

92 determine whether symptomatic menopausal transition is independently linked to stroke.

\section{Materials and Methods}

95 Data Source

96 Data were retrieved from the National Health Insurance Research Database in Taiwan 
97 (NHIRD); this was implemented in 1995 and covers $>99 \%$ of the population (approximately 23

98 million people). A database containing 1 million patients randomly selected from the NHIRD in

992005 and longitudinally linked with NHIRD from 1996 to 2013 was anonymously accessed for

100 research. The comprehensive healthcare information maintained in the database included the date

101 of birth, sex, area of residence, income, ambulatory care, inpatient services, prescription drugs,

102 medical procedures, and diagnostic codes. The International Classification of Diseases, Ninth

103 Revision, Clinical Modification (ICD-9-CM) codes, which have been shown to have high

104 accuracy and validity, were used to identify the diseases (Cheng, et al., 2011; Cheng, et al., 2014;

$105 \mathrm{Yu}$, et al., 2012). This study was exempt from a full ethical review and was approved by the

106 institutional review board (IRB) of the Changhua Christian Hospital (approval number 190522).

107 The requirement for consent was waived by the IRB.

108 Study population

109 We used a 4-year look-back period (1996-1999) to identify subjects with newly diagnosed

110 symptomatic menopausal transition by excluding pre-existing diagnosis (Fig. 1). Symptomatic

111 menopausal transition was defined by at least three records made by gynecologists within a 1-

112 year period (ICD-9-CM code 627.2: symptomatic menopausal transition). Subjects who were

113 diagnosed with symptomatic menopausal transition during the look-back period were excluded

114 from this study. We identified 457996 subjects, including 41516 with newly diagnosed 
115 symptomatic menopausal transition and 416480 without symptomatic menopause, from the

116 NHIRD between January 1, 1996 and December 31, 2013. The index date of the symptomatic

117 menopausal transition group was defined as the first date of the symptomatic menopausal

118 transition diagnosis; this date was also assigned to the matched comparison participants as the

119 date of entry into the study. The exclusion criteria for the study and comparison groups were as

120 follows: (1) age, $<45$ or $>100$ years, (2) history of breast cancer before index date (breast cancer

121 treatments can cause menopausal symptoms or premature menopause) (Cusack et al., 2013), (3)

122 history of oophorectomy before index date, (4) history of stroke before index date, (5) patients

123 who did not survive or were followed up $<30$ days (a short exposure time to symptomatic

124 menopausal transition may not be sufficient to cause an incident stroke), and (6) patients who

125 were not matched to the comparison group. Finally, 22058 women with newly diagnosed

126 symptomatic menopausal transition with no history of stroke were enrolled between January 1,

1272000 and December 31, 2013. Additionally, 22058 comparison participants were selected by

128 propensity score matching. Both groups were followed up until the date of death, developing

129 stroke, withdrawal from the National Health Insurance, or the end of 2013. The mean follow-up

130 period was 8.5 years (standard deviation 4.7 years).

131 Outcome measures and relevant variables

132 The primary outcome was the development of stroke following the index date. The stroke 
133 (ICD-9-CM codes: 430-438) and comorbidities were diagnosed by at least three records. These

134 diagnostic codes were either from outpatient claims or principal diagnoses of hospitalization

135 records. Potential confounders were included based on previous literature and the possible

136 relationships between them and stroke were examined; these included hypertension,

137 hyperlipidemia, diabetes mellitus, obesity, chronic kidney disease, coronary artery disease,

138 congestive heart failure, chronic obstructive pulmonary disease, dysrhythmia, peripheral artery

139 occlusive disease, and long-term medications (Tsai, et al., 2016; Chen, et al., 2015). We also

140 included additional covariates such as hormone replacement therapy (HRT) and duration of

141 symptomatic menopause. The proxy measures of symptomatic menopause duration were based

142 on the length of time period between the first and last symptomatic menopause-related visits

143 reported by gynecologists.

144 Statistical analysis

145 Propensity score matching was performed to balance the distributions of measured covariates

146 (all variables in Table 1) in the symptomatic menopausal transition and comparison groups. The

147 propensity score was calculated using non-parsimonious multivariable logistic regression, and all

148 variables in Table 1 (age, monthly income, hypertension, hyperlipidemia, diabetes mellitus,

149 obesity, chronic kidney disease, coronary artery disease, congestive heart failure, chronic

150 obstructive pulmonary disease, dysrhythmia, peripheral artery occlusive disease, Charlson's 
151 comorbidity index score, clinic visit frequency, antihypertensives, antidiabetic agents, statins,

152 antiplatelets, aspirin, warfarin, and hormone replacement therapy) were included for each

153 patient. Comparison participants were matched on propensity score (nearest-neighbor algorithm

154 with a caliper of 0.1 standard deviation) at a 1:1 ratio (Austin, 2008; Kuo, et al., 2015; Weng, et

155 al., 2017). The standardized difference (StD) was used to measure the imbalance of all clinical

156 characteristics between the two groups. A StD $\geq 0.1$ indicated a significant imbalance between

157 the two groups (Austin, 2008). The cumulative incidence of stroke over time was estimated using

158 the cumulative incidence function curves. Deaths prior to the development of stroke were

159 considered as competing risks. Therefore, we conducted the competing risks survival analysis

160 using the Fine and Gray subdistribution hazards model. The association of symptomatic

161 menopausal transition with subsequent development of stroke was reported as subhazard ratios

162 (SHRs) and 95\% confidence intervals (CIs). Statistical analyses were conducted using SAS 9.4

163 software (SAS Institute Inc). A two-sided $P$ value $<0.05$ was considered statistically significant.

164

165 Results

166 Table 1 shows the baseline characteristics of the two groups. A total of 44116 women (22058

167 women diagnosed with symptomatic menopausal transition and 22058 matched comparison 
168 participants) with no history of stroke were enrolled in the study. The mean age of the enrolled

169 subjects was $52.6 \pm 7.4$ years. The age, monthly income, comorbidities, Charlson's comorbidity

170 index score, clinic visit frequency, and long-term medications were balanced between the two

171 groups, except a predilection for HRT in the symptomatic menopausal transition group.

172

173 Risk of stroke after perimenopausal transition

174 During the follow-up period, 1184 women (5.37\%) of the comparison group and 2274

$175(10.31 \%)$ of the symptomatic menopausal transition group developed stroke (Fig. 1). The

176 incident rates of stroke were 8.57 (95\% CI, 8.08-9.06 per 1000 person-years) and 11.17 (95\%

177 CI, 10.71-11.63 per 1000 person-years) in the comparison and symptomatic menopausal transition groups, respectively (Table 2). The cumulative incidence of stroke was higher in the symptomatic menopausal transition group than in the comparison group (Fig. 2). The crude risk of stroke in the symptomatic menopausal transition group was statistically significantly higher compared to the comparison group (SHR, 1.31;95\% CI, 1.22-1.41; $P<0.001)$. Additionally,

182 two multivariable models were used to adjust the risk of subsequent stroke. After adjusting for

183 all confounders in Table 1 (Model 2, Table 2) and the full adjustment model (Model 3, Table 2),

184 the risk of subsequent stroke remained consistently higher in the symptomatic menopausal

185 transition group than that of the comparison group (SHR, 1.37, 95\% CI, 1.27-1.48, $P<0.001$ 
186 and SHR, 1.30, 95\% CI, 1.21-1.40, $P<0.001$, respectively).

\section{Risk of stroke by age, comorbidities, and HRT}

188 The SHR for subsequent stroke was statistically significantly higher in women with

189 symptomatic menopause compared to comparison participants; this was the case for young $(<50$

190 years), middle-aged (50-64 years), and elderly ( $\geq 65$ years) women. However, the interaction

191 between symptomatic menopausal transition and age category was statistically significant

$192\left(P_{\text {interaction }}<0.001\right.$, Table 3$)$. The risk of developing stroke tended to decrease with increasing age

193 (SHRs from 1.42 to 1.29 to 1.19 ). Additionally, there was a statistically significant interaction

194 between symptomatic menopausal transition and comorbidity $\left(P_{\text {interaction }}<0.001\right.$, Table 3$)$.

195 Symptomatic menopausal women without comorbid condition had a higher risk of developing

196 stroke than those with any comorbidity (SHRs, 1.41 vs. 1.14). However, the interaction between

197 the use of HRT and stroke was not statistically significant $\left(P_{\text {interaction }}=0.66\right.$, Table 3$)$ though the

198 SHR for stroke was only statistically significantly higher in symptomatic menopausal women

199 without HRT compared to comparison participants.

200 Subgroup analyses

201 The risk of subsequent stroke was further estimated throughout the baseline clinical

202 characteristics, including individual comorbidities and long-term medications (Figure 3). We

203 found that there was no statistically significant interaction between symptomatic menopausal 
204 transition and all subgroups except hypertension and use of antihypertensives $\left(P_{\text {interaction }}<0.001\right.$

205 and 0.003 , respectively). The risk of stroke was further increased in symptomatic menopausal

206 women without hypertension or without the use of antihypertensives (Figure 3).

207

208 Duration of symptomatic menopausal transition and stroke

209 We estimated the association of the duration of symptomatic menopausal transition with the

210 development of subsequent stroke. The longer duration of symptomatic menopausal transition

211 was associated with higher risk of stroke (Table 4).

212

213 Discussion

214 The present study is the first and largest study to investigate the association between

215 symptomatic menopausal transition and subsequent development of stroke during up to 14 years

216 of follow-up. In this prognostic model, symptomatic menopausal transition has added

217 statistically significantly prognostic power for stroke to standard risk factors. Women with

218 symptomatic menopausal transition had a $30 \%$ increased risk of subsequent stroke compared to

219 matched comparison participants. The effect of symptomatic menopause on stroke was

220 consistent across all subgroups except age, comorbidity, hypertension, and the use of 
221 antihypertensives. Moreover, this effect was durable and increased when the duration of

222 menopausal transition lasted longer.

223 Previous studies have shown that there is a possible link between stroke and symptomatic

224 menopausal transition. Women experiencing vasomotor symptoms tend to have lower circulating

225 estradiol levels and other adverse cardiovascular risk factors such as obesity, smoking, and

226 psychosocial problems (Gast, et al., 2010). Among them, low socioeconomic status, depression,

227 and anxiety have the closest relationship between these factors and cardiovascular diseases

228 (Thurston, et al., 2012). Additionally, dysregulation of adipocyte-derived hormones, especially

229 the higher circulating leptin levels, lower adiponectin levels, and higher leptin-to-adiponectin

230 ratios, was reported in women transitioning through menopause (Ben, et al., 2011). A recent

231 study also showed that the profile of adipokines (leptin levels, adiponectin levels, and leptin-to-

232 adiponectin ratios) was closely related to the severity of vasomotor symptoms (Huang, et al.,

233 2017). Leptin, for example, is a widely investigated adipokine and its production is proportional

234 to the adipose tissue mass and its secretion is pulsatile with the characteristics of diurnal rhythm.

235 The serum leptin level is highest at night and lowest in the morning; as the leptin level rises, it

236 increases the appetite via its effects on the brain, resulting in weight gain and obesity (Kelesidis,

237 et al., 2010). Moreover, both menopause and low levels of estrogens can cause systemic

238 inflammation and neuroinflammation (Au, et al., 2016). Therefore, a low estrogen level, 
239 dysregulation of adipokine production, high BMI, and proinflammatory status may be potential

240 underlying pathogenic factors for stroke in women with symptomatic menopausal transition.

241 In the present study, the risk of stroke was especially high in women with early onset of

242 symptomatic menopause, not using antihypertensives, in the absence of comorbidity, and without

243 hypertension. Lisabeth et al. reported that natural menopause before age 42 was associated with a

244 higher risk of ischemic stroke (Lisabeth, et al., 2009). Another study also suggested that early

245 menopause is associated with an increased risk of ischemic stroke (Rocca, et al., 2012). Early

246 menopause may expose women to longer duration of systemic- or neuro-inflammation

247 contributing to a higher risk of subsequent stroke. We also observed an interaction between

248 symptomatic menopausal transition and comorbidity whereby the risk of stroke was highest in

249 women without any comorbidity and the effect of symptomatic menopausal transition on stroke

250 was significantly reduced in women with a comorbid condition. Furthermore, since blood

251 pressure control is one of the most important risk factors for stroke, especially ischemic stroke in

252 women, we evaluated the interaction between symptomatic menopausal transition and

253 hypertension or use of antihypertensives (Gorgui, et al., 2014). Our results revealed that the risk

254 of stroke was much higher in women without or without control of hypertension. Because

255 hypertension is already a strong risk factor for stroke, the impact of symptomatic menopausal

256 transition on subsequent stroke becomes smaller in the hypertension or with antihypertensives 
257 subgroups than in the non-hypertension or without antihypertensives subgroups. Our findings

258 suggest that these subgroups are at the highest risk for stroke and that the prevention of

259 subsequent stroke should be considered in addition to traditional management of symptomatic

260 menopause.

261 Vasomotor symptoms are some of the major problems leading middle-aged women to seek

262 medical advice and treatment for symptomatic menopause. Although HRT plays a key role in

263 treating symptomatic menopausal transition (Erlik, Meldrum, \& Judd, 1982; Huang, et al., 2008),

264 HRT has been reported to increase the risk of breast cancer (Russo \& Russo, 2006), cerebral

265 (Rossouw, et al., 2002) or cardiac vascular events (Wilson, Garrison, \& Castelli, 1985), and new-

266 onset atrial fibrillation (Tsai, et al., 2016; Bretler, et al., 2012). We found that the risk of stroke

267 remained higher in women with symptomatic menopausal transition after adjustment for HRT.

268 Furthermore, there was no statistically significant interaction between symptomatic menopausal

269 transition and HRT.

270 The strength of our study is its large sample size. A large sample size is more likely to

271 produce sufficiently narrow CIs. In this case, the narrow CIs of our results (e.g., 95\% CI of SHR,

272 1.21-1.40; Table 2) indicate adequate precision. We did not conduct ex ante power calculations

273 because our study utilized medical claims retrospectively retrieved from the NHIRD. However,

274 the retrospective power of this study calculated using an observed effect size was determined 
275 entirely by the $P$ value which was already observed. Thus, post hoc sample size calculation or

276 power analysis provides no additional information beyond the effect size. Additionally, the

277 number of events observed may be important when modeling survival data. Previous studies

278 have shown that 10-50 events per variable may be necessary to assure accurate estimation of

279 regression coefficients, standard errors, and confidence intervals (Austin et al., 2017; Peduzzi et

280 al., 1995). A 14-year follow-up was more than appropriate to detect stroke events in middle-aged

281 women (3458 stroke events were observed during the study period). However, there were some

282 limitations to this study. First, the NHIRD did not include information relevant to stroke such as

283 smoking history, BMI, physical activity, daily salt intake, blood pressure, and blood glucose and

284 lipid profiles. These unmeasured covariates could have an impact on the outcome, even after

285 balancing the baseline clinical characteristics with propensity score matching. Second, the

286 diagnosis of symptomatic menopausal transition was mainly based on the ICD-9-CM codes; this

287 could lead to the underestimation of cases with less severe symptoms. However, the risk of

288 stroke may be underestimated if the cases were misclassified as matched comparison

289 participants. Third, we cannot exclude the "healthy user bias"; patients who seek medical advice

290 might be compliant with healthy lifestyles or treatments that could reduce the occurrence of

291 adverse events (Brookhart, et al., 2007). Finally, since most of the population in Taiwan are Han

292 Chinese, further studies are required to clarify whether these findings can be generalized to other 
293 ethnicities.

294

\section{Conclusion}

296 This large-scale long-term cohort study demonstrated that symptomatic menopausal transition

297 was statistically significantly associated with an increased risk of subsequent stroke. Women

298 with early menopausal transition, without comorbid condition, without hypertension, or using

299 antihypertensives are at a higher risk of stroke. In order to fully elucidate whether stroke

300 prevention improves cerebrovascular outcomes in the care of symptomatic menopausal transition

301 further studies are required.

302

303 Conflict of interest disclosure

304 None.

305 Acknowledgements

306 None.

307 Funding

308 This study was funded by grants 106-CCH-IRP-022, 107-CCH-NFP-002, and 107-CCH-HCR-

309031 from the Changhua Christian Hospital Research Foundation. The funding agency played no 
310 role in the study design, data collection, analysis, data interpretation, or in the preparation of the

311 manuscript, and the decision to publish.

312

\section{References}

314 Au A, Feher A, McPhee L, Jessa A, Oh S, Einstein G. 2016. Estrogens, inflammation and

315 cognition.Frontiers in Neuroendocrinology 40:87-100.

316 Austin PC. 2008. A critical appraisal of propensity-score matching in the medical literature

317 between 1996 and 2003. Statistics in Medicine 27(12):2037-49.

318 Austin PC, Allignol A, Fine JP. 2017. The number of primary events per variable affects

319 estimation of the subdistribution hazard competing risks model. J Clin Epidemiol 83:75-84.

320 Ben Ali S, Jemaa R, Ftouhi B, Kallel A, Feki M, Slimene H, Kaabachi N. 2011. Relationship of

321 plasma leptin and adiponectin concentrations with menopausal status in Tunisian women.

322 Cytokine 56(2):338-42.

323 Bretler DM, Hansen PR, Lindhardsen J, Ahlehoff O, Andersson C, Jensen TB, Raunsø J, Torp-

324 Pedersen C, Gislason GH. 2012. Hormone replacement therapy and risk of new-onset atrial

325 fibrillation after myocardial infarction-a nationwide cohort study. PloS One 7(12):e51580.

326 Brookhart MA, Patrick AR, Dormuth C, Avorn J, Shrank W, Cadarette SM, Solomon DH. 2007.

327 Adherence to lipid-lowering therapy and the use of preventive health services: an investigation 
328 of the healthy user effect. The American Journal of Epidemiology 166(3):348-54.

329 Cheng CL, Kao YH, Lin SJ, Lee CH, Lai ML. 2011. Validation of the National Health Insurance

330 Research Database with ischemic stroke cases in Taiwan. Pharmacoepidemiology and Drug

331 Safety 20(3):236-42.

332 Cheng CL, Lee CH, Chen PS, Li YH, Lin SJ, Yang YH. 2014. Validation of acute myocardial

333 infarction cases in the national health insurance research database in Taiwan. Journal of

334 epidemiology 24(6):500-7.

335 Chen YH, Hsieh TF, Lee CC, Wu MJ, Fu YC. 2015. Estrogen therapy and ischemic stroke in

336 women with diabetes aged over 55 years: a nation-wide prospective population-based study in

337 Taiwan. PloS One 10(12):e0144910.

338 Cusack L, Brennan M, Baber R, Boyle F. 2013. Menopausal symptoms in breast cancer

339 survivors: management update. Br J Gen Pract 63:51-2.

340 Erlik Y, Meldrum DR, Judd HL. 1982. Estrogen levels in postmenopausal women with hot

341 flashes. Obstetrics and Gynecology 59(4):403-7.

342 Gallicchio L, Miller SR, Kiefer J, Greene T, Zacur HA, Flaws JA. 2014. Change in body mass

343 index, weight, and hot flashes: a longitudinal analysis from the midlife women's health study.

344 Journal of Women's Health 23(3):231-7.

345 Gast GC, Samsioe GN, Grobbee DE, Nilsson PM, van der Schouw YT. 2010. Vasomotor 
346 symptoms, estradiol levels and cardiovascular risk profile in women. Maturitas 66(3):285-90.

347 Gorgui J, Gorshkov M, Khan N, Daskalopoulou SS. 2014. Hypertension as a risk factor for

348 ischemic stroke in women. The Canadian Journal of Cardiology 30(7):774-82.

349 Hankey GJ. 2017. Stroke. Lancet 389(10069):641-54.

350 Huang AJ, Grady D, Jacoby VL, Blackwell TL, Bauer DC, Sawaya GF. 2008. Persistent hot

351 flushes in older postmenopausal women. Archives of Internal Medicine 168(8):840-6.

352 Huang WY, Chang CC, Chen DR, Kor CT, Chen TY, Wu HM. 2017. Circulating leptin and

353 adiponectin are associated with insulin resistance in healthy postmenopausal women with hot

354 flashes. PLoS One 12(4):e0176430.

355 Kelesidis T, Kelesidis I, Chou S, Mantzoros CS. 2010. Narrative review: the role of leptin in

356 human physiology: emerging clinical applications. Annals of Internal Medicine 152(2):93-100.

357 Kuo KL, Hung SC, Liu JS, Chang YK, Hsu CC, Tarng DC. 2015. Add-on protective effect of

358 pentoxifylline in advanced chronic kidney disease treated with renin-angiotensin-aldosterone

359 system blockade - a nationwide database analysis. Scientific Reports 5:17150.

360 Lisabeth LD, Beiser AS, Brown DL, Murabito JM, Kelly-Hayes M, Wolf PA. 2009. Age at

361 natural menopause and risk of ischemic stroke: the Framingham heart study. Stroke 40(4):1044-

3629.

363 Ohayon MM. 2006. Severe hot flashes are associated with chronic insomnia. Archives of Internal 
364 Medicine 166(12):1262-8.

365 Peduzzi P, Concato J, Feinstein AR, Holford TR. 1995. Importance of events per independent

366 variable in proportional hazards regression analysis. II. Accuracy and precision of regression

367 estimates. J Clin Epidemiol 48:1503-1510.

368 Rocca WA, Grossardt BR, Miller VM, Shuster LT, Brown RD Jr. 2012. Premature menopause or

369 early menopause and risk of ischemic stroke. Menopause 19(3):272-7.

370 Rossouw JE, Anderson GL, Prentice RL, LaCroix AZ, Kooperberg C, Stefanick ML, Jackson

371 RD, Beresford SA, Howard BV, Johnson KC, Kotchen JM, Ockene J; Writing Group for the

372 Women's Health Initiative Investigators. 2002. Risks and benefits of estrogen plus progestin in

373 healthy postmenopausal women: principal results from the Women's Health Initiative

374 randomized controlled trial. Jama 288(3):321-33.

375 Rossouw JE, Prentice RL, Manson JE, Wu L, Barad D, Barnabei VM, Ko M, LaCroix AZ,

376 Margolis KL, Stefanick ML. 2007. Postmenopausal hormone therapy and risk of cardiovascular

377 disease by age and years since menopause. Jama 297(13):1465-77.

378 Russo J, Russo IH. 2006. The role of estrogen in the initiation of breast cancer. The Journal of

379 Steroid Biochemistry and Molecular Biology 102(1-5):89-96.

380 Santoro N, Epperson CN, Mathews SB. 2015. Menopausal symptoms and their management.

381 Endocrinology and Metabolism Clinics of North America 44(3):497-515. 
382 Thurston RC, El Khoudary SR, Sutton-Tyrrell K, Crandall CJ, Gold EB, Sternfeld B, Joffe H,

383 Selzer F, Matthews KA. 2012. Vasomotor symptoms and lipid profiles in women transitioning

384 through menopause. Obstetrics and Gynecology 119(4):753-61.

385 Thurston RC, Chang Y, Barinas-Mitchell E, Jennings JR, von Känel R, Landsittel DP, Matthews

386 KA. 2017. Physiologically assessed hot flashes and endothelial function among midlife women.

387 Menopause 24(8):886-93.

388 Tsai CF, Thomas B, Sudlow CL. 2013. Neurology 81(3):264-72.

389 Tsai WC, Haung YB, Kuo HF, Tang WH, Hsu PC, Su HM, Lin TH, Chu CS, Jhuo SJ, Lee KT,

390 Sheu SH, Chen CY, Wu MT, Lai WT. 2016. Hormone replacement therapy and risk of atrial

391 fibrillation in Taiwanese menopause women: A nationwide cohort study. Scientific Reports

$3926: 24132$.

393 U.S. Census B. Women in the United States. 2005.

394 https://factfinder.census.gov/faces/tableservices/jsf/pages/productview.xhtml?pid=ACS_10_1YR

395 _S0101\&prodType=table. Accessed May 1, 2019.

396 Weng SC, Wu CL, Kor CT, Chiu PF, Wu MJ, Chang CC, Tarng DC. Migraine and subsequent

397 chronic kidney disease risk: a nationwide population-based cohort study. BMJ Open

398 7(12):e018483.

399 Wilson PW, Garrison RJ, Castelli WP. 1985. Postmenopausal estrogen use, cigarette smoking, 
400 and cardiovascular morbidity in women over 50. The Framingham Study. The New England

401 Journal of Medicine 313(17):1038-43.

402 Yu YB, Gau JP, Liu CY, Yang MH, Chiang SC, Hsu HC, Hong YC, Hsiao LT, Liu JH, Chiou

403 TJ, Chen PM, Lee TS, Chou LF, Tzeng CH, Chen TJ. 2012. A nation-wide analysis of venous

404 thromboembolism in 497,180 cancer patients with the development and validation of a risk-

405 stratification scoring system. Thrombosis and Haemostasis 108(2):225-35. 


\section{Figure 1}

Study flowchart illustrating the patient selection process and primary outcome.

After exclusion of noneligible subjects, 22058 women with newly diagnosed symptomatic menopausal transition with no history of stroke were enrolled between January 1, 2000 and December 31, 2013. Additionally, 22058 matched comparison participants were selected by propensity score matching at a 1:1 ratio. Furthermore, 1184 women $(5.37 \%)$ of the comparison group and $2274(10.31 \%)$ of the symptomatic menopausal transition group developed stroke during the follow-up period. The propensity score was calculated by using all characteristic variables of each subject, including age, monthly income, hypertension, hyperlipidemia, diabetes mellitus, obesity, chronic kidney disease, coronary artery disease, congestive heart failure, chronic obstructive pulmonary disease, dysrhythmia, peripheral artery occlusive disease, Charlson's comorbidity index score, clinic visit frequency, antihypertensives, antidiabetic agents, statins, antiplatelets, aspirin, warfarin, and hormone replacement therapy. NHIRD, National Health Insurance Research Database in Taiwan. 


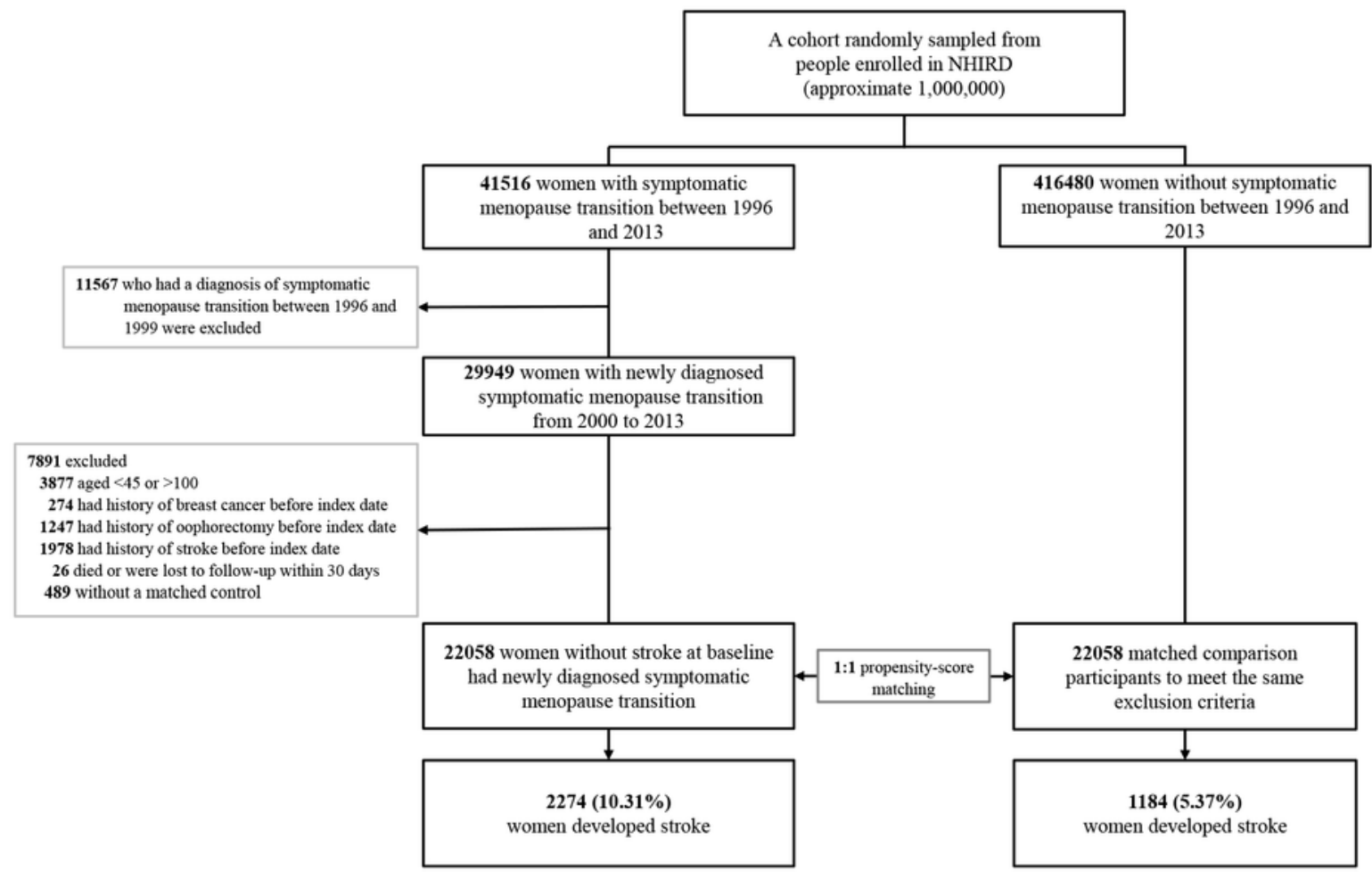


Figure 2

Cumulative incidence function curves with 95\% confidence intervals for the risk of subsequent stroke between the two groups.

The cumulative incidence of stroke was significantly higher in the symptomatic menopausal transition group (SMT) than in the comparison group.

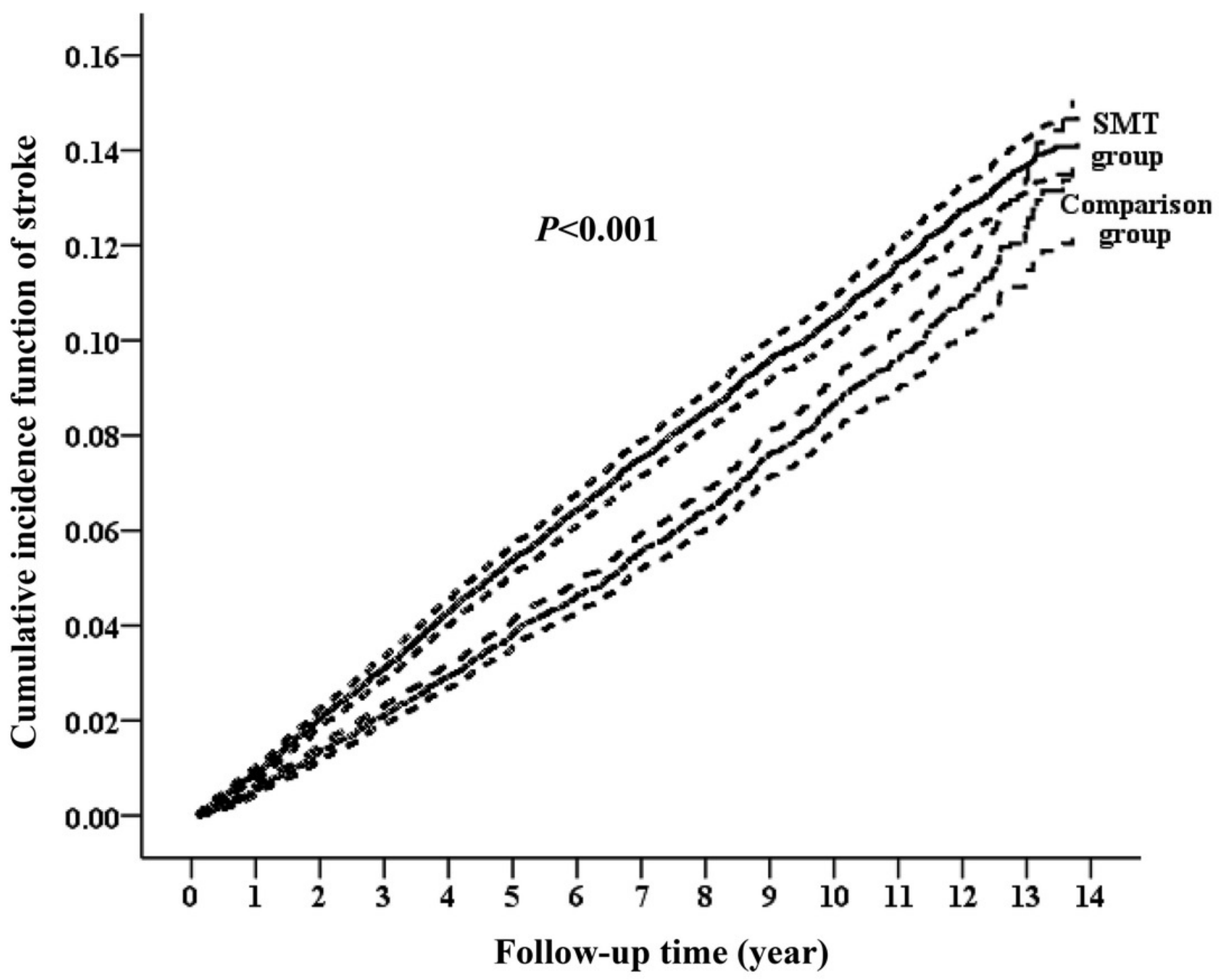




\section{Figure 3}

Subgroup analyses.

The risk of subsequent stroke was consistent across all subgroups except hypertension and use of antihypertensives. aSHR, adjusted subhazard ratio; CAD, coronary artery disease; CHF, congestive heart failure; $\mathrm{Cl}$, confidence interval; $\mathrm{CKD}$, chronic kidney disease; COPD, chronic obstructive pulmonary disease. 


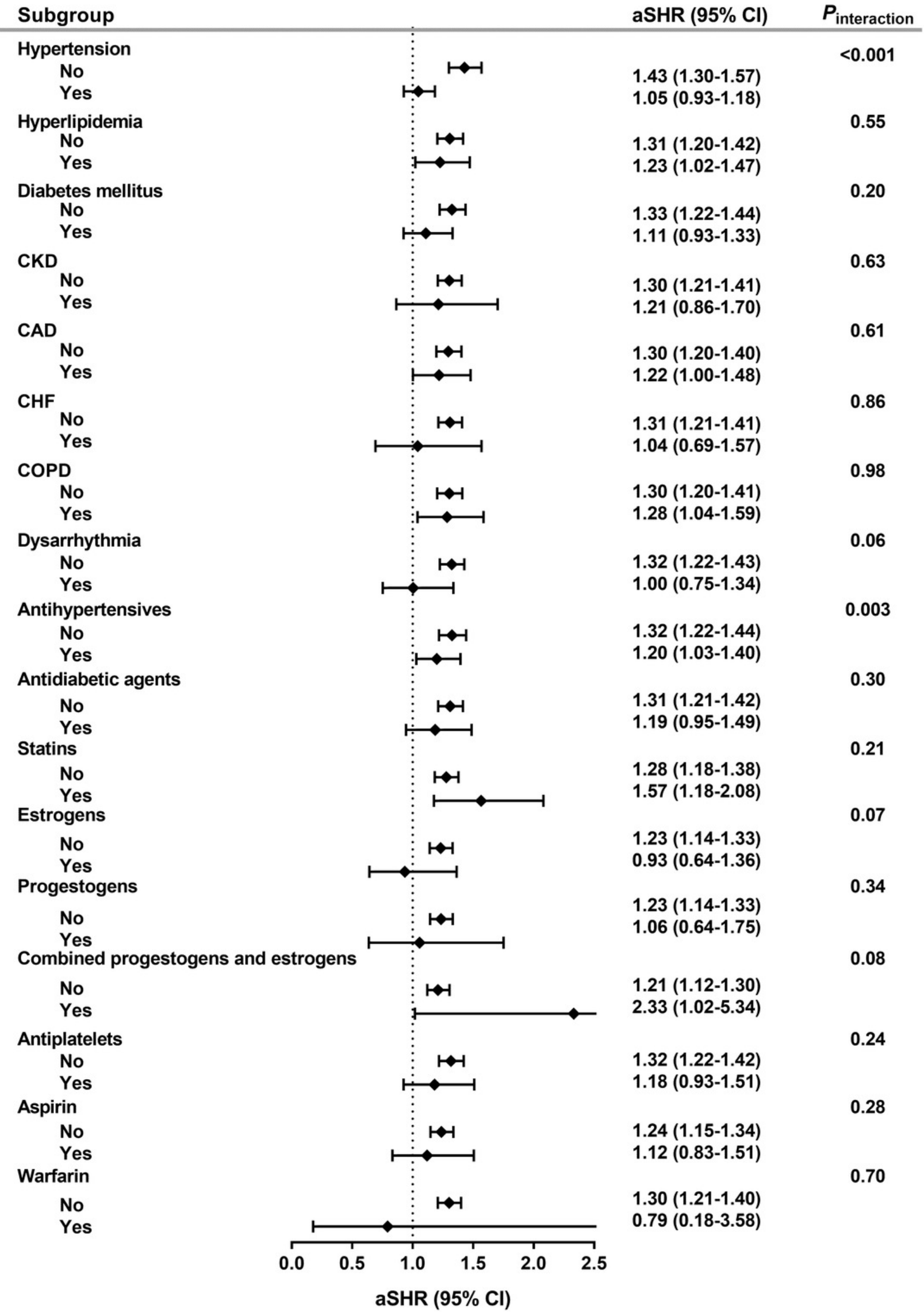


Table $\mathbf{1}$ (on next page)

Demographics and clinical characteristics at baseline 
1 Table 1. Demographics and clinical characteristics at baseline

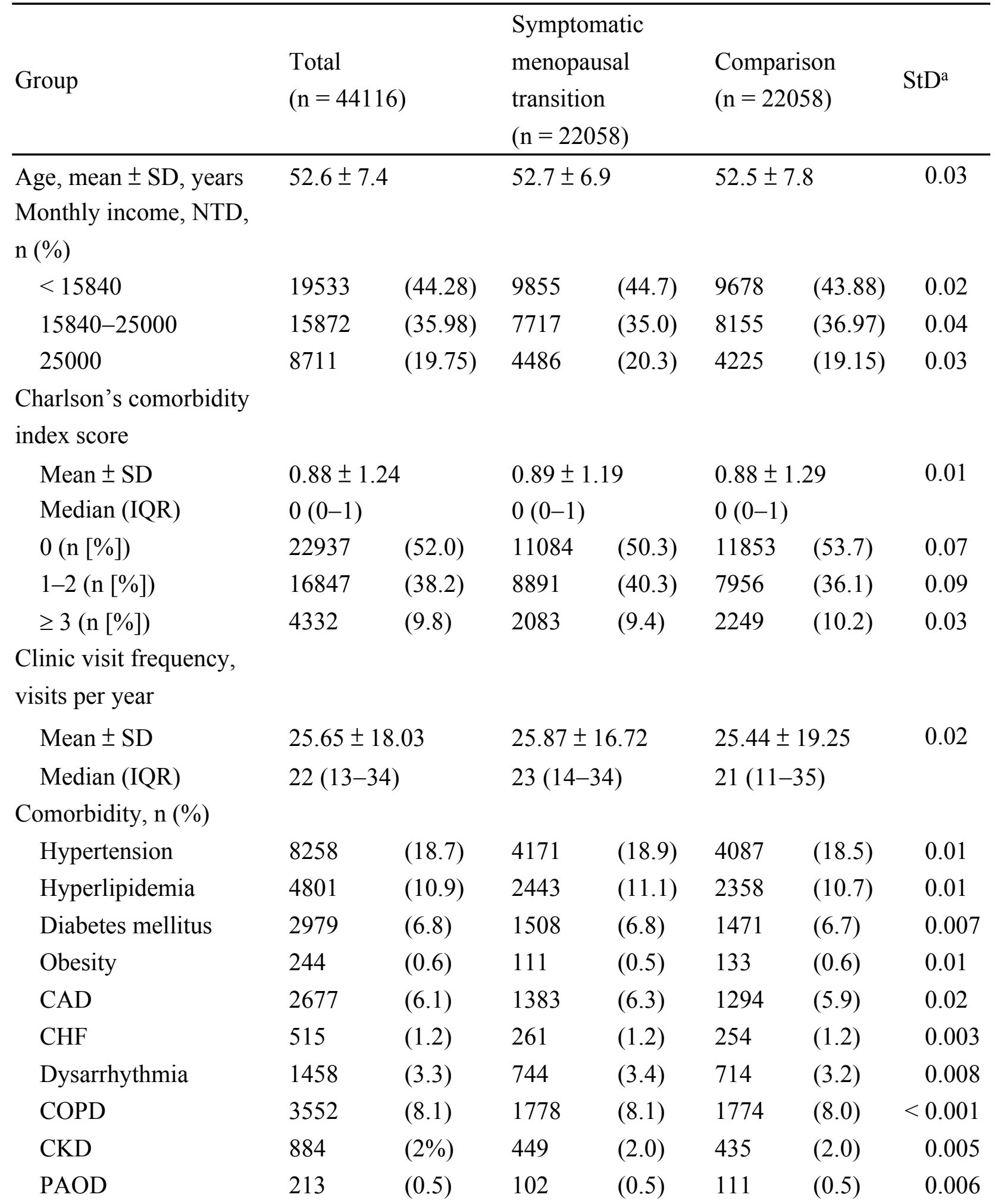

Long-term use

medications $^{\mathrm{b}}, \mathrm{n}(\%)$ 


\begin{tabular}{llllllll} 
Antihypertensives & 5353 & $(12.1)$ & 2695 & $(12.2)$ & 2658 & $(12.1)$ & 0.005 \\
Antidiabetic agents & 1710 & $(3.9)$ & 863 & $(3.9)$ & 847 & $(3.8)$ & 0.004 \\
$\begin{array}{l}\text { Statins } \\
\text { Hormone }\end{array}$ & 1467 & $(3.3)$ & 753 & $(3.4)$ & 714 & $(3.2)$ & 0.01 \\
$\begin{array}{l}\text { replacement therapy } \\
\quad \text { Estrogens }\end{array}$ & 5383 & $(12.2)$ & 4916 & $(22.3)$ & 467 & $(2.1)$ & 0.65 \\
$\quad \begin{array}{l}\text { Progestogens } \\
\text { Combined }\end{array}$ & 2863 & $(6.5)$ & 2591 & $(11.8)$ & 272 & $(1.2)$ & 0.44 \\
$\quad \begin{array}{l}\text { progestogens and } \\
\text { estrogens }\end{array}$ & 2362 & $(4.7)$ & 1845 & $(8.4)$ & 206 & $(0.9)$ & 0.36 \\
$\begin{array}{l}\text { Antiplatelets } \\
\quad \text { Aspirin }\end{array}$ & 1607 & $(3.6)$ & 818 & $(3.7)$ & 789 & $(3.6)$ & 0.007 \\
$\begin{array}{l}\text { Warfarin } \\
\text { Propensity score (mean }\end{array}$ & 1169 & $(2.7)$ & 561 & $(2.5)$ & 608 & $(2.8)$ & 0.01 \\
SD) & 75 & $(0.2)$ & 36 & $(0.2)$ & 39 & $(0.2)$ & 0.003 \\
\hline
\end{tabular}

2 astandardized difference (StD) of greater than 0.1 is considered important imbalance.

3 bDefined as drug prescription for at least 3 consecutive months.

4 Abbreviations: $\mathrm{CAD}$, coronary artery disease; $\mathrm{CHF}$, congestive heart failure; $\mathrm{CKD}$, chronic

5 kidney disease; COPD, chronic obstructive pulmonary disease; IQR, interquartile range; NTD,

6 new Taiwan dollars; PAOD, peripheral artery occlusive disease; SD, standard deviation; StD,

7 standardized difference.

8 
Table 2 (on next page)

Incidence and risk of stroke in patients with symptomatic menopausal transition and their matched subjects 
Table 2. Incidence and risk of stroke in patients with symptomatic menopausal transition and their matched subjects

\begin{tabular}{|c|c|c|c|c|c|c|c|c|c|}
\hline \multirow[b]{2}{*}{ Group } & \multirow[b]{2}{*}{ Event } & \multirow[b]{2}{*}{$\mathrm{PY}^{\mathrm{a}}$} & \multirow[b]{2}{*}{ Incidence $^{\mathrm{b}}$} & \multicolumn{2}{|l|}{ Model 1} & \multicolumn{2}{|l|}{ Model 2} & \multicolumn{2}{|l|}{ Model 3} \\
\hline & & & & $\begin{array}{l}\mathrm{cSHR}^{\mathrm{c}} \\
(95 \% \mathrm{CI})\end{array}$ & $P$-value & $\begin{array}{l}\mathrm{aSHR}^{\mathrm{c}} \\
(95 \% \mathrm{CI})\end{array}$ & $P$-value & $\begin{array}{l}\mathrm{aSHR}^{\mathrm{c}} \\
(95 \% \mathrm{CI})\end{array}$ & $P$-value \\
\hline Comparison & 1184 & 138138.72 & $\begin{array}{l}8.57(8.08- \\
9.06)\end{array}$ & Referenc & & Referenc & & Referen & \\
\hline $\begin{array}{l}\text { Symptomatic } \\
\text { menopausal } \\
\text { transition }\end{array}$ & 2274 & 203586.37 & $\begin{array}{l}11.17 \\
(10.71- \\
11.63)\end{array}$ & $\begin{array}{l}1.31(1.22- \\
1.41)\end{array}$ & $<0.001$ & $\begin{array}{l}1.37(1.27- \\
1.48)\end{array}$ & $<0.001$ & $\begin{array}{l}1.30(1.21- \\
1.40)\end{array}$ & $<0.001$ \\
\hline
\end{tabular}

2 Abbreviations: aSHR, adjusted subhazard ratio; cSHR, crude subhazard ratio; CI, confidence interval; PY, person-years.

3 Model 1: crude hazard ratio compared with the propensity-score matched comparison subjects.

4 Model 2: adjusted for all variables listed in Table 1.

5 Model 3: adjusted for all variables listed in Table 1, as well as comorbidities and medications considered as time-dependent

6 covariates.

7 aPY, person-years.

8 bPer 1000 person-years.

9 'Death before developing stroke was considered a competing risk. 


\section{Table 3(on next page)}

Risk of stroke in patients with symptomatic menopausal transition and their matched subjects regarding the age of onset, comorbidity, and hormone replacement therapy 
1 Table 3. Risk of stroke in patients with symptomatic menopausal transition and their matched subjects regarding the age of

2 onset, comorbidity, and hormone replacement therapy

\begin{tabular}{|c|c|c|c|c|c|c|c|c|c|}
\hline \multirow[t]{2}{*}{ Subgroup } & \multicolumn{2}{|c|}{ Comparison } & \multicolumn{2}{|c|}{$\begin{array}{l}\text { Symptomatic } \\
\text { menopausal } \\
\text { transition }\end{array}$} & \multicolumn{5}{|c|}{ Symptomatic menopausal transition vs. comparison } \\
\hline & $\mathrm{n}$ & Event & $\mathrm{n}$ & Event & $\begin{array}{c}\text { Model } 1 \\
\text { aSHR }(95 \% \text { CI })^{\mathrm{a}, \mathrm{b}}\end{array}$ & $P$-value & $\begin{array}{c}\text { Model } 2 \\
\operatorname{aSHR}(95 \% \mathrm{CI})^{\mathrm{b}, \mathrm{c}}\end{array}$ & $P$-value & $P_{\text {interaction }}{ }^{\mathrm{d}}$ \\
\hline Age, years & & & & & & & & & $<0.001$ \\
\hline$<50$ & 10534 & 237 & 8712 & 490 & $1.55(1.31-1.83)$ & $<0.001$ & $1.42(1.21-1.67)$ & $<0.001$ & \\
\hline $50-64$ & 9403 & 527 & 11647 & 1289 & $1.30(1.16-1.45)$ & $<0.001$ & $1.29(1.16-1.43)$ & $<0.001$ & \\
\hline$\geq 65$ & 2121 & 420 & 1699 & 495 & $1.20(1.03-1.39)$ & 0.02 & $1.19(1.03-1.37)$ & 0.02 & \\
\hline Comorbidity & & & & & & & & & $<0.001$ \\
\hline 0 & 15409 & 515 & 14348 & 984 & $1.58(1.41-1.78)$ & $<0.001$ & $1.41(1.26-1.58)$ & $<0.001$ & \\
\hline$\geq 1$ & 6649 & 669 & 7710 & 1290 & $1.19(1.07-1.32)$ & 0.001 & $1.14(1.03-1.26)$ & 0.01 & \\
\hline $\begin{array}{l}\text { Hormone replacement } \\
\text { therapy }\end{array}$ & & & & & & & & & 0.66 \\
\hline No & 21591 & 1146 & 17142 & 1637 & $1.35(1.25-1.47)$ & $<0.001$ & $1.27(1.17-1.38)$ & $<0.001$ & \\
\hline Yes & 467 & 38 & 4916 & 637 & $1.28(0.92-1.79)$ & 0.15 & $1.25(0.90-1.75)$ & 0.19 & \\
\hline
\end{tabular}

3 Abbreviations: aSHR, adjusted subhazard ratio; CI, confidence interval.

4 aAdjusted for all variables listed in Table 1.

5 b Death before developing stroke was considered a competing risk.

6 cAdjusted for all variables listed in Table 1, as well as comorbidities and medications considered as time-dependent covariates.

$7 \quad \mathrm{~d} P$-values for interactions were obtained from Model 2. 


\section{Table 4 (on next page)}

Table 4. Risk of stroke in patients with symptomatic menopausal transition compared with the comparison group regarding the duration of symptomatic menopause 
1 Table 4. Risk of stroke in patients with symptomatic menopausal transition compared with the comparison group regarding

2 the duration of symptomatic menopause

\begin{tabular}{lllll}
\hline $\begin{array}{l}\text { Duration of symptomatic } \\
\text { menopause (years) }\end{array}$ & aSHR $(95 \% \mathrm{CI})^{\mathrm{a}, \mathrm{b}}$ & $P$-value & aSHR $(95 \% \mathrm{CI})^{\mathrm{b}, \mathrm{c}}$ & $P$-value \\
\hline 0 & Reference & - & Reference & - \\
$0-1.4$ & $1.20(1.09-1.33)$ & $<0.001$ & $1.18(1.07-1.29)$ & 0.001 \\
$1.4-4.8$ & $1.29(1.17-1.43)$ & $<0.001$ & $1.26(1.14-1.39)$ & $<0.001$ \\
$>4.8$ & $1.41(1.28-1.55)$ & $<0.001$ & $1.33(1.21-1.45)$ & $<0.001$ \\
$P$ for trend & & $<0.001$ & & $<0.001$ \\
\hline
\end{tabular}

3 Abbreviations: aSHR, adjusted subhazard ratio; CI, confidence interval.

4 aAdjusted for all variables listed in Table 1.

5 bDeath before developing stroke was considered a competing risk.

6 cAdjusted for all variables listed in Table 1, as well as comorbidities and medications considered as time-dependent covariates. 